SHORT COMMUNICATION

\title{
Morphological aspects of coconut anther culture derived structures
}

\author{
P.I.P. Perera ${ }^{1,5^{*}}$, D.M.D. Yakandawala ${ }^{2,5}$, J.L. Verdeil ${ }^{3}$, V. Hocher ${ }^{4}$ and L. K. Weerakoon ${ }^{1}$ \\ Tissue Culture Division, Coconut Research Institute, Bandirippuwa Estate, Lunuwila. \\ 2 Department of Botany, Faculty of Science, University of Peradeniya, Peradeniya. \\ 3 Institute for Research and Development (IRD), UMR 1098 BEPC, IRD, BP 64501 - 911 Avenue Agropolis, 34394 Montpellier Cedex 1, France. \\ ${ }^{4}$ CIRAD, TA40/02 Avenue Agropolis, 34398 Montpellier Cedex 5, France. \\ ${ }_{5}^{5}$ Postgraduate Institute of Science, P.O. Box 25, University of Peradeniya, Peradeniya.
}

\begin{abstract}
Anthers excised from male flowers of an adult coconut palm of Sri Lanka Tall cultivar were used to produce plants via androgenesis. Morphological aspects of the antherderived structures and their plant regeneration pathways were studied. Under the culture conditions employed, both calli and embryo-mediated plant regeneration were observed. Two types of embryos with different germination patterns were identified-one with a germination point and the other type with a blunt haustorium without a prominent germination point which converted into germinated embryos. Three types of calli (embryogenic compact calli, embryogenic friable calli and non-embryogenic fast-growing calli) were also produced and the most commonly identified type was embryogenic compact calli that give rise to somatic embryos. Secondary somatic embryos were produced in both direct embryo and callus mediated embryogenesis. Normal plants with a single shoot were observed in low frequencies while weak plantlets with multiple shoot were observed in abundance. Under similar culture conditions, some plantlets showed vigorous growth whereas the majority had a slow growth rate.
\end{abstract}

Keywords: Androgenesis, anther culture, callus, coconut (Cocos nucifera L.), embryo, morphology.

\section{INTRODUCTION}

Production of double haploid plants through tissue culture technology has a great potential in producing homozygous lines for hybrid plant production. The haploids are induced by triggering the normal developmental process of the gametes into the sporophytic pathway ${ }^{1}$. Previous attempts at producing doubled haploid coconut plants by other research groups have resulted in limited success ${ }^{2-6}$. For the first time, coconut haploid/doubled haploid plants were produced consistently from the research carried out at the Coconut Research Institute, Lunuwila ${ }^{7}$. This achievement will have a tremendous impact on generating new varieties through the coconut breeding programmes that are greatly affected by high heterozygosity, resulting from the allogamous nature of the palm.

In vitro culture conditions (for inducing androgenesis of cultured anthers), such as pollen developmental stage, pre-treatment, culture medium and anther density have been optimised ${ }^{7}$. Growth regulators are vital for induction of calli/embryos in cultured anthers. 2-4 dichlorophenoxyacetic acid (2,4-D) and 6-( $\square, \square$ dimethylallylamino)-purine (2iP) alone could give rise to a high frequency of anther-derived calli/ embryos. However, $\square$-naphthyl acetic acide (NAA) in combination with 2,4-D enhanced the frequency of calli/ embryo production ${ }^{8}$. The frequency of plant regeneration ultimately decides the efficiency of the in vitro protocol developed. Prolonged culture in liquid medium resulted in low regeneration frequency and high vitrification. Regeneration frequency could be improved with the use of a solidified medium (unpublished data). Histological studies showed that calli and embryos originated from microspores ${ }^{7}$. The analysis of anther-derived structures by flow cytometry and Simple Sequence Repeats (SSR) markers revealed that they were haploids/doubled haploids ${ }^{9}$ indicating that the protocol developed was in correct order. Genotype of the mother palm greatly affected the induction of androgenesis and out of six palms tested only one palm responded with androgenesis ${ }^{10}$. However, androgenesis induction and plant regeneration 
was consistent from the anthers collected from the selected palm.

In any tissue culture protocol, plant regeneration involves the development of different stages with specific morphologies. Identification of the morphological characteristics at different developmental stages is an important criterion to overcome the practical difficulties when assessing the regeneration pathway. Studies on molecular and biochemical markers associated with embryogenic competence have been conducted with various crops. Molecular analysis of somatic embryogenesis has been done mostly by comparing genes and proteins expressed in embryogenic and nonembryogenic cells in different stages of embryogenesis ${ }^{11}$. Although the reliability of these techniques is high, the procedures are destructive and time consuming. The quickest way to assess a culture is by the use of morphological features at different developmental stages. Thus, the objective of this paper was to determine the morphological aspects of coconut anther culture that can be used to assess the developmental process of antherderived plantlets.

\section{METHODS AND MATERIALS}

Androgenesis induction and plant regeneration was performed according to the protocol described in previous studies $^{7}$. Anthers 3 wks prior to the splitting stage were excised from male flowers of an adult coconut palm of the Sri Lanka Tall cultivar. A heat shock at $38^{\circ} \mathrm{C}$ was given for $6 \mathrm{~d}$ prior to culture. Modified Eeuwens $\mathrm{Y}_{3}$ medium ${ }^{12}$ supplemented with $100 \mu \mathrm{M}$ 2,4-D, $0.1 \%$ (w/v) activated charcoal (acid washed, BDH, UK) and $9 \%$ (w/v) sucrose was used for androgenesis induction. After adjusting the $\mathrm{pH}$ to 5.8 the medium was solidified with $0.3 \%(\mathrm{w} / \mathrm{v})$ phytagel.

Hundred anthers were cultured in 10 Petri plates (100 x $10 \mathrm{~mm} ; 10$ anthers/plate), each containing $25 \mathrm{~mL}$ of culture medium. Cultures were maintained and observed over a 9 month period. The cultures were observed at $2 \mathrm{wk}$ intervals. After measuring the diameter of the embryos and calli, they were subcultured in the somatic embryo induction medium (modified Eeuwens $\mathrm{Y}_{3}$ solid medium with $66 \mu \mathrm{M}$ 2,4-D) for 4 wks followed by somatic embryo maturation medium (modified Eeuwens $\mathrm{Y}_{3}$ solid medium without any hormones) for 4 wks. The embryogenic structures were then transferred and maintained in a germination medium [modified Eeuwens $\mathrm{Y}_{3}$ solid medium supplemented with $5 \mu \mathrm{M}$ N-6-benzyladenine (BAP), $0.1 \mu \mathrm{M}$ 2,4-D and $0.35 \mu \mathrm{M}$ Gibberellic acid $\left(\mathrm{GA}_{3}\right)$ ]. After embryo germination, the cultures were exposed to light $[16 \mathrm{~h}$ photoperiod; photosynthetically active radiation (PAR); $25 \mu \mathrm{molm}^{-2} \mathrm{~s}^{-1}$. All morphological features of the antherderived structures were recorded by the naked eye and the different plant regeneration pathways were studied.

\section{RESULTS AND DISCUSSION}

Pollen embryogenesis was successfully induced in coconut anthers confirming previous studies? ${ }^{7}$. Both direct and callus-mediated embryogenesis were observed consistently under the culture conditions employed.

During the incubation period, a distinct change in size or shape of anthers was not observed. However, anthers turned brown after about ten weeks of culture initiation. Similar observations were made in anther cultures of Cassia siamea ${ }^{13}$ and Vitis latifolia ${ }^{14}$. Swelling of anthers has also been observed prior to androgenic induction in some crop species including Fagopyrum esculentum Monench (buckwheat) ${ }^{15}$, Cassia siamea ${ }^{13}$ and Cucumis sativus ${ }^{16}$.

When excising anthers from male flowers of coconut, complete detachment of anther from the filament was not possible in some cases. In anthers cultured along with the filament, pollen embryogenesis was observed but callus development on the filaments was not observed. The fresh appearance of the filaments diminished with time and eventually they turned brown. Previous studies ${ }^{17}$ reported that remnant filament tissues significantly decreased embryo production in broccoli (Brassica oleracea var. italica) by lowering the number of anthers forming embryos. However, when plants with high androgenic capacity were used, high frequency of embryo formation was achieved even with the filament attached ${ }^{17}$. It has been reported that filament-derived calli emerged at very early stages of culturing when compared to pollen-derived calli in Camellia sinensis, Vitis latifolia and Brassica oleracea var. italica ${ }^{14,17,18}$. Furthermore, differences exist between filament-derived and pollen-derived calli with regard to their morphology and colour ${ }^{19}$. Callus formation from the anther wall has been observed in Cassia siamea ${ }^{13}$ and Citrus aurantifolia ${ }^{20}$. No callus formation was observed from the anther wall in this study.

Induction of embryogenic structures could be observed after three months of culture initiation and continued up to eight months indicating that the potential for pollen embryogenesis lasts for a considerable period of time ${ }^{7}$. After eight months, the embryogenic potential of the anthers diminished and this could probably be due to the loss of pollen viability. The peak response 
of anthers was observed five months after culture initiation ${ }^{7}$. Formation of both embryos and calli could be observed under the same culture conditions and similar observations have been made in anther cultures of cucumber ${ }^{16}$ and eggplant ${ }^{21}$. However, the frequency of embryos was much higher than that of the calli.

The embryos could be distinguished on the surface of the anther. They emerged through the grove of the anther lobe (Figure 1a). Embryos at different developmental stages could be observed on the same anther (Figure 1b). The maturity of embryos was determined based on their shape and colour. The mature embryos were white and opaque with heart or round shape whereas the immature embryos were translucent and round in shape (Figure 1b). The size of mature embryos ranged from 1 $6 \mathrm{~mm}$. Embryos were attached to the anther lobe by a tiny connector-like structure that was easily detached even by a slight movement. The embryos had the tendency to fall off readily, indicating the absence of any physical connection with the somatic tissues of the anther wall ${ }^{7}$.

If the concentration of auxin in the culture medium is high enough, instead of proceeding to the next stage of its ontogeny, a somatic embryo may give rise to new

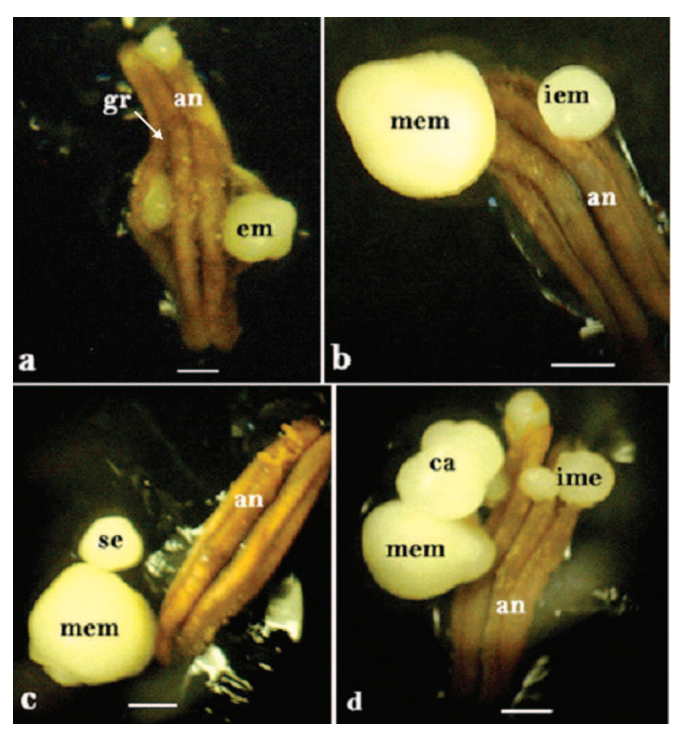

Figure 1: Formation of embryos in cultured coconut anthers a. Embryos emerging by breaking through the grove (gr) of the anther (an) (Bar=1.2 mm). Note the three embryos at different maturity stages. b. Mature embryo (mem) (opaque) and immature embryo (iem) (translucent) on the same anther (Bar=1 mm). c. Secondary embryos (se) produced by the embryos derived from the cultured anther (Bar=1.2 $\mathrm{mm}$ ). d. Formation of a compact callus (ca) by douching the emerging embryo with the androgenesis induction medium (Bar=1.2 mm). somatic embryos, a process called secondary or repetitive embryogenesis ${ }^{22}$. Occasionally, secondary embryos were derived from primary embryos (Figure 1c) when they were maintained in the same androgenesis induction medium without subculturing. It has been reported that such cycles of repetitive embryogenesis can be broken by the removal or reduction of auxin in the culture medium ${ }^{22}$. In the present study, the secondary embryos produced germinated. In contrast to this observation, Guo et al., ${ }^{23}$ observed that in Timothy, the secondary embryos were produced after subculturing the compact primary embryos from induction medium to the regeneration medium. Secondary embryogenesis has also been observed in Vitis latifolia ${ }^{14}$.

In addition to embryos, different types of calli were also produced under the same culture conditions. Calli were produced directly from cultured anthers and also from embryos when they were in direct contact with the medium after falling off from the anther. This could be due to exposure of embryos to a high concentration of 2,4-D present in the medium. It could also be due to the anther wall restricting the supply of growth regulators to the developing embryo stimulating it to become a fully matured embryo. Development of embryogenic friable

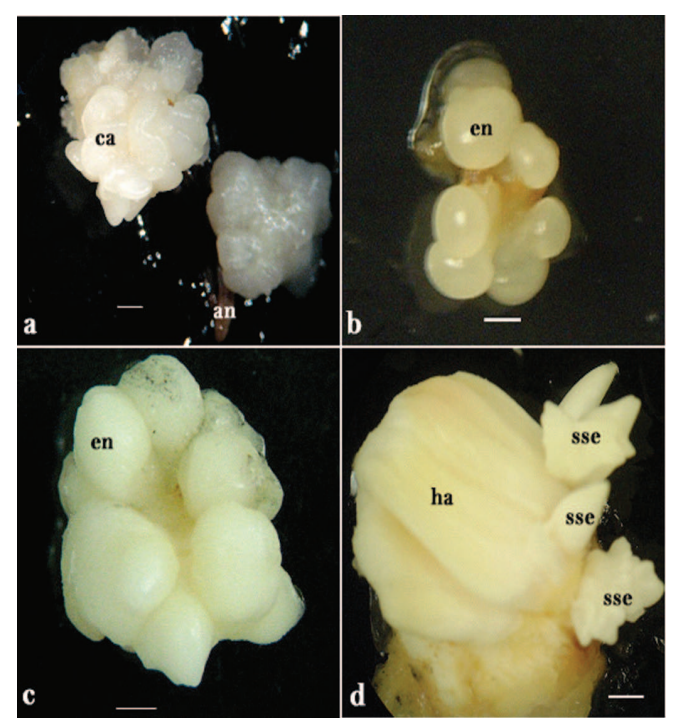

Figure 2: Somatic embryogenesis of anther derived calli of coconut. a. Well developed compact callus (Bar=1.5 mm). b. A friable callus (Bar=1 mm). The embryogenic nodules (en) are loosely attached to each other. c. Somatic embryo developed from embryogenic nodules (Bar=1.7 $\mathrm{mm}$ ). d. Formation of secondary somatic embryos (sse) from the haustorial tissue (ha) of the primary somatic embryo. (Bar=3 mm). 
calli with loosely attached embryogenic nodules was rare whereas compact calli (Figure 2a) were more common in cultures. This type of compact calli have been observed in cultured anthers of the carob tree ${ }^{24}$. Pollen-derived calli with similar morphology have been described in tea ${ }^{18}$. Non-embryogenic fast growing calli were also produced in some cultures at a very low frequency.

Anthers bearing both embryos and calli could be observed (Figure 1d) in some cultures. According to previous studies ${ }^{25}$, direct embryogenesis is more desirable due to higher incidence of green plant regeneration. Lack of proliferation of somatic cells in the cultured coconut anthers and the absence of physical contact with the anther wall indicated that the embryos and calli have arisen from microspores.

Subculturing of calli as well as immature embryos into the medium with reduced levels of 2,4-D is important to induce embryogenesis. The reduction or removal of 2,4-D from the regeneration medium has shown to be essential for optimal plant regeneration ${ }^{26,27}$. Removal of growth regulators at the next subculture stage allows the embryo to become fully matured with the formation of shoot and root poles. Previous studies ${ }^{28}$ have reported that the elimination of plant growth regulators from the culture medium stimulates regeneration of plants. In anther culture of wheat, it has been observed that androgenic plant regeneration from calli was accompanied by the formation of a conspicuous extracellular matrix surface network (ECMSN) around the induced callus cells and young embryo-like structures and its structural arrangement changed during culture ${ }^{29}$. However, calli-derived somatic embryos in the present study had low regeneration capacity and further investigations are necessary to improve the efficiency of plant regeneration.

The calli, consisting of a translucent mass of globules (Figures 2a, 2b) gave rise to somatic embryos (Figure 2c) when they were transferred to embryo maturation medium (modified Eeuwens $\mathrm{Y}_{3}$ medium devoid of growth regulators). In the carob tree, the calli transferred to media without any auxin, failed to produce shoots or embryos and the multi-cellular structures and the pro-embryos showed no further development ${ }^{24}$. The formation of secondary somatic embryos (Figure $2 \mathrm{~d}$ ) could be observed in some cultures. Some of these secondary somatic embryos germinated and gave rise to shoots. However, the frequency of secondary embryo formation was very low, possibly due to the lack of an appropriate medium for differentiation as seen in the soybean anther culture ${ }^{30}$.
In the mature embryo, polarisation into haustorium and radical end could be seen in embryo maturation medium. Two types of embryos with different germination patterns could be observed. In most of the mature embryos, a germination point could be seen as a depression on the surface (Figure 3a). The shoot emerged through this point (Figure $3 \mathrm{~b}$ ) giving rise to a fully germinated embryo (Figure 3c) later on. The other type of embryo had a blunt haustorium without a germination point (Figure $3 \mathrm{~d}$ ). In these embryos, the shoot emerged by splitting of the haustorial tissue (Figure 3e), which later gave rise to a completely germinated embryo (Figure $3 \mathrm{f}$ ). These shoots were subcultured into germination medium containing $5 \mu \mathrm{M}$ BAP, $0.1 \mu \mathrm{M} 2,4-\mathrm{D}$ and $0.35 \mu \mathrm{M} \mathrm{GA}_{3}$ for further development.

Certain irregularities were observed during the plant regeneration process. Developmental abnormalities (i.e. multiple shoot meristems and fused embryos) were observed in some of the embryos produced. Further

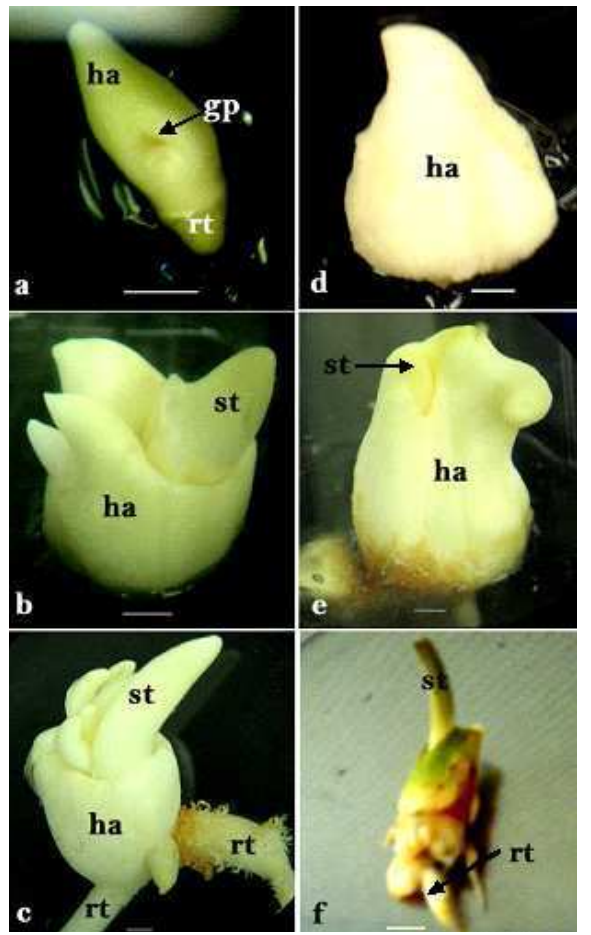

Figure 3: Maturation and conversion of anther derived embryos. a. A mature somatic embryo showing the germination point (gp) $(\mathrm{Bar}=1 \mathrm{~mm})$. b. A converted embryo (Bar=2 mm). Note that the shoot (st) is emerging through the germination point. c. Further development of the shoot of the converted embryo (Bar=2.7 mm). d. A mature embryo with blunt ended haustorial tissue (ha) (Bar=2mm). Note that it does not contain the germination point e. Embryo germination (Bar=10 mm). Note the shoot emerging by splitting through the haustoriual tissue f. A germinated embryo with both shoot and roots $(\mathrm{rt})(\mathrm{Bar}=10 \mathrm{~mm})$. 


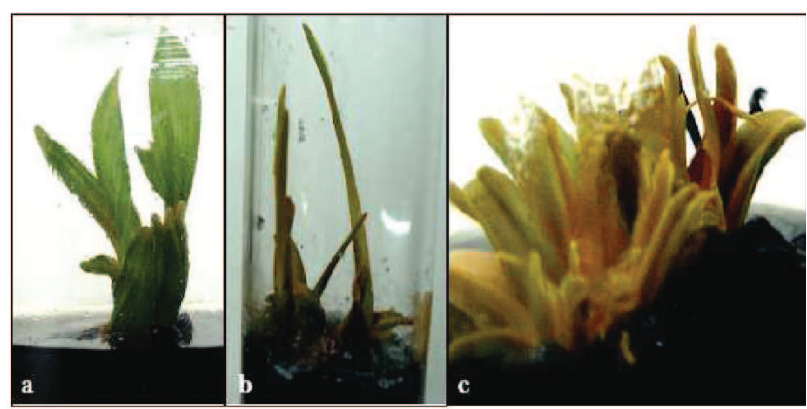

Figure 4: Anther derived plantlets a. single shoot. b. double shoot. c. multiple shoot

development of many embryos was arrested due to these abnormalities. Some of the somatic embryos did not germinate properly. Even after repeated subculture into germination medium, many of these embryos failed to develop proper shoots.

The germinated embryos developed into complete plantlets by continuous subculturing into the germination medium. Among the plantlets produced, morphological variations could be observed based on the number of meristematic points. Initially, all of them developed with a single shoot point (Figure 4a) but some plantlets produced either double shoots (Figure 4b) or multiple shoots (Figure 4c) later.

Multiple shoot regeneration has also been observed commonly in flax anther culture ${ }^{31}$. The potential for production of multiple shoots could be induced by subculturing them into the medium containing $\mathrm{GA}_{3}$. The plantlets with single or double shoots were of good quality and had a healthy appearance. However, the shoots in a cluster seemed to be weak and developed very slowly. Further research needs to be directed towards regeneration of plants since the success of an anther culture protocol depends on efficient plant regeneration.

Under similar culture conditions, some plantlets showed vigorous growth, whereas the majority showed very slow growth. Differences in various traits (leaf shape, leaf angle, etc.) among the different haploid lines (as shown in Figure 4) support the hypothesis that these lines originated from different microspores which were genetically different. Thus, it may be possible to identify different genotypes among tall coconut haploid plants as observed in tall fescue haploids ${ }^{32}$.

The present study indicated the different aspects of the anther culture protocol developed for coconut. Under the culture conditions employed, both calli and embryomediated plant regeneration could be observed. Two types of embryos with different germination patterns could be observed-one with a germination point and the other type with a blunt haustorium without a germination point. Plants with a single germination point, as well as plants with multiple shoots were also observed. Under similar culture conditions, some plantlets showed vigorous growth whereas the majority showed a very slow growth. All the structures that develop at different stages of the protocol were repeatedly observed in the ongoing experiments with coconut anther cultures.

\section{Acknowledgement}

We are thankful to Mr. I. Wikramananda for his assistance in digital photography at the initial stage of the present study.

\section{References}

1. Ascough G.D., Bakos F., Balazs E., Barnabas B. \& Van Staden J. (2006). Screening South African wheat germplasm for androgenic competence. South African Journal of Botany 72:40-45.

2. Iyer R.D. (1981). Embryo and tissue culture for crop improvement, especially of perennials, germplasm conservation and exchange-relevance to developing countries. (Ed. A.N. Rao), Proceedings of the COSTED Symposium on Tissue Culture of Economically Important Plants. pp. 219-230. National University of Singapore, Singapore.

3. Iyer R.D. \& Raina S.K. (1972). The early ontogeny of embryos and callus from pollen and subsequent organogenesis in anther cultures of Datura metal and rice. Planta 104:146-156.

4. Kovoor A. (1981). Palm tissue culture: state of art and its application to the coconut. $5^{\text {th }}$ Session of the FAO Technical Working Party on Coconut Production and Protection pp. 37-52. Food and Agriculture Organization, Rome.

5. Thanh-Tuyen N.T. \& De Guzman E.V. (1983). Pollen developmental stages for coconut anther culture. Philippine Journal of Biology 12(1-2):135-144.

6. Monfort S. (1985). Androgenesis of coconut: embryos from anther culture. Zeitschrift für Pflanzenzüchtng 94:251-254.

7. Perera P.I.P., Hocher V., Verdeil J-L., Bandupriya H.D.D., Yakandawala D.M.D. \& Weerakoon L.K. (2008) Androgenic potential in coconut (Cocos nucifera L.). Plant Cell Tissue and Organ Culture 92:293-302.

8. Perera P.I.P., Yakandawala D.M.D., Hocher V., Verdeil J-L. \& Weerakoon L.K. (2009). Effect of growth regulators on microspore embryogenesis in coconut anthers. Plant Cell Tissue and Organ Culture 96:171-180.

9. Perera P.I.P., Perera L., Hocher V., Verdeil J-L., Yakandawala D.M.D. \& Weerakoon L.K. (2008). Use of SSR markers to determine the anther-derived homozygous lines in coconut. Plant Cell Reports 27:1697-1703. 
10. Perera P.I.P., Yakandawala D.M.D., Hocher V., Verdeil J-L. \& Weerakoon L.K. (2008). Variations among anthers and their orientation during in vitro culture for inducing androgenesis in coconut. (Eds. N.P.A.D. Nainanayake \& J.M.D.T. Everard) Export Competitiveness Through Quality Improvement $2^{\text {nd }}$ Symposium on Plantation Crop Research, pp 104-110. Colombo.

11. Rao K.S. (1996). Embryogenesis in flowering plantsrecent approaches and prospects. Journal of Bioscience 21(6): 827-841.

12. Karunaratne S., Kurukulaarachchi C. \& Gamage C. (1985). A report on the culture of embryos of dwarf coconut, Cocos nucifera L. var. nana, in vitro. Cocos 3:1-8.

13. Gharyal P.K., Rashid A. \& Maheshwari S.C. (1983). Androgenic response from cultured anthers of a leguminous tree Cassia siamea Lam. Protoplasma 118:91-93.

14. Salunkhe C.K., Rao P.S. \& Mhatre M. (1999). Plantlet regeneration via somatic embryogenesis in anther callus of Vitis latifolia L. Plant Cell Reports 18:670-673.

15. Bohanec B., Neskovic M. \& Vujicic R. (1993). Anther culture and androgenetic plant regeneration in buckwheat (Fagopyrum esculentum Moench). Plant Cell, Tissue and Organ Culture 35:259-266.

16. Ashok-Kumar H.G. \& Murthy H.N. (2004). Effect of sugars and amino acids on androgenesis of Cucumis sativus. Plant Cell, Tissue and Organ Culture 78:201-208

17. Arnison P.G., Donaldson P., Ho L.C.C. \& Keller W.A. (1990). The influence of various physiological parameters on anther culture of broccoli (Brassica oleracea var. italica). Plant Cell, Tissue and Organ Culture 20:147155.

18. Raina S.K. \& Iyre R.D. (1983). Multicelled pollen proembryos and callus formation in tea anther culture. Journal of Plantation Crops (Supplement): 63-67.

19. Shimada T. (1981). Haploid plants regenerated from the pollen callus of wheat (Triticum aestivum L.). Japan Journal of Genetics 56:581-588.

20. Chaturvedi H.C. \& Sharma A.K. (1985). Production of androgenic plants of Citrus aurantifolia. Journal of Plant Physiology 119:473-477.

21. Rizza F., Mennella G., Collonnier C., Sihachakr D., Kashyap V., Rajam M.V., Prestera M. \& Rotino G.L. (2002). Androgenic doubled haploids from somatic hybrids between Solanum melongena and S. aethiopicum group gilo as a source of resistance to Fusarium oxysporum $\mathrm{f}$. sp. melongenae. Plant Cell Reports 20:1022-1032.

22. ParrottW.A., Merkle S.A. \& Williams E.G. (1991). Somatic embryogenesis: potential for use in propagation and gene transfer systems. In: Advanced Methods in Plant Breeding and Biotechnology. (Ed. D.R. Murray). pp. 158-200. CAB International, Wallingford Oxon OX108DE, UK.

23. Guo Y-D., Sewon P. \& Pulli S. (1999). Improved embryogenesis from anther culture and plant regeneration in Timothy. Plant Cell, Tissue and Organ Culture 57:85-93.

24. Custodio L., Carneiro M.F. \& Romano A. (2005). Microsporogenesis and anther culture in carob tree (Ceratonia siliqua L.). Scientia Horticulturae 104(1): 65-77.

25. Mukhambetzhanov S.K. (1997). Culture of nonfertilized female gametophytes in vitro. Plant Cell, Tissue and Organ Culture 48:111-119.

26. Ball S.T., Zhou H. \& Konzak C.F. (1993). Influence of 2,4-D, IAA and duration of callus induction in anther culture of spring wheat. Plant Science 90:195-200.

27. Zheng M.Y. \& Konzak C.F. (1999). Effect of 2,4 dichlorophenoxyacetic acid on callus induction and plant regeneration in anther culture of wheat (Triticum asetivum L.). Plant Cell Reports 19:69-73.

28. Trejo-Tapia G., Amaya U.M., Morales G.S., Sanchez A.D.J., Bonfil B.M., Rodriguez-Monroy M. \& JimenezAparicio (2002). The effects of cold-pretreatment, auxins and carbon source on anther culture of rice. Plant Cell, Tissue and Organ Culture 71:41-46.

29. Konieczny R., Bohdanowicz J., Czaplicki A.Z. \& Przywara L. (2005). Extracellular matrix surface network during plant regeneration in wheat anther culture. Plant Cell, Tissue and Organ Culture 83:201-208.

30. Rodrigues L.R., Oliveira J.M.S., Mariath J.E.A., Iranco L.B. \& Bodanese-Zanettini M.H. (2005). Anther culture and cold treatment of floral buds increased symmetrical and extra nuclei frequencies in soybean pollen grains. Plant Cell, Tissue and Organ Culture 81:101-104.

31. Chen Y., Hausner G., Kenaschuk E., Procunier D., Dribnenki P. \& Penner G. (1998). Identification of microspore-derived plants in anther culture of flax (Linum usitatissimum L.) using molecular markers. Plant Cell Reports 18:44-48.

32. Kasperbauer M.J. \& Eizenga G.C. (1985). Tall Fescue doubled haploids via tissue culture and plant regeneration. Crop Science 25:1091-1095. 